\title{
A Linear-Time Algorithm \\ for Concave One-Dimensional Dynamic Programming
}

Zvi Galil

Kunsoo Park

CUCS-469-89 


\title{
A Linear-Time Algorithm for Concave One-Dimensional Dynamic Programming*
}

\author{
Zvi Galil ${ }^{1,2}$ and Kunsoo Park ${ }^{1}$ \\ ${ }^{1}$ Department of Computer Science, Columbia University, New York, NY 10027 \\ 2 Department of Computer Science, Tel-Aviv University, Tel-Aviv, Israel
}

Keywords: Dynamic programming, quadrangle inequality, total monotonicity

The one-dimensional dynamic programming problem is defined as follows: given a real-valued function $w(i, j)$ for integers $0 \leq i \leq j \leq n$ and $E[0]$, compute

$$
E[j]=\min _{0 \leq i<j}\{D[i]+u(i, j)\} . \quad \text { for } 1 \leq j \leq n,
$$

where $D[i]$ is computed from $E[i]$ in constant time. The least weight subsequence problem [4] is a special case of the problem where $D[i]=E[i]$. The modified edit distance problem [3], which arises in molecular biology, geology, and speech recognition, can be decomposed into $2 n$ copies of the problem.

Let $A$ be an $n \times m$ matrix. $A[i, j]$ denotes the element in the $i$ th row and the $j$ th column. $A\left[i: i^{\prime}, j: j^{\prime}\right]$ denotes the submatrix of $A$ that is the intersection of rows $i, i+1, \ldots, i^{\prime}$ and columns $j, j+1, \ldots, j^{\prime}$. We say that the cost function $w$ is concave if it satisfies the quadrangle inequality $[i]$

$$
w(a, c)+w(b, d) \leq w(b, c)+w(a, d), \quad \text { for } a \leq b \leq c \leq d .
$$

In the concave one-dimensional dynamic programming problem $w$ is concave as defined above. A condition closely related to the quadrangle inequality was introduced by Aggarwal et al. [1] An $n \times m$ matrix $A$ is totally monotone if for all $a<b$ and $c<d$,

$$
A[a, c]>A[b, c] \quad \Longrightarrow \quad A[a, d]>A[b, d]
$$

Let $r(j)$ be the smallest row index such that $A[r(j), j]$ is the minimum value in column $j$. Then total monotonicity implies

$$
r(1) \leq r(2) \leq \cdots \leq r(m)
$$

That is, the minimum row indices are nondecreasing. We say that an element $A[i . j]$ is dead if $i \neq r(j)$. A submatrix of $A$ is dead if all of its elements are dead. Note that for $a \leq b \leq c \leq d$, the quadrangle inequality implies total monotonicity. but the converse is not true. Aggarwal et al. [1] show that the row maxima of a totally monotone $n \times m$ matrix $A$ can be found in $O(n+m)$ time if $A[i, j]$ for any $i, j$ can be computed in constant time. Their algorithm is easily adapted to find the column minima. We will refer to their algorithm as the SMAWK algorithm.

Let $B[i, j]=D[i]+w(i, j)$ for $0 \leq i<j \leq n$. We say that $B[i, j]$ is available if $D[i]$ is known and therefore $B[i, j]$ can be computed in constant time. Then the problem is to find the column minima in the upper triangular matrix $B$ with the restriction that $B[i, j]$ is avalable only after the column minima for columns $1,2, \ldots, i$ have been found. It is easy to see that when $w$ satisfies the quadrangle inequality, $B$ also satisfies the quadrangle inequality. For the concave problem Hirschberg and Larmore [4] and later Galil and Giancarlo [3] gave $O(n \log n)$ algorithms using queues. Wilber [6] proposed an $O(n)$ time algorithm when $D[i]=E[i]$. However, his algorithm does not work if the availability of matrix $B$ must be obeyed, which happens when many copies

* Work supported in part by NSF Grants CCR-86-05353 and CCR-88-14977 


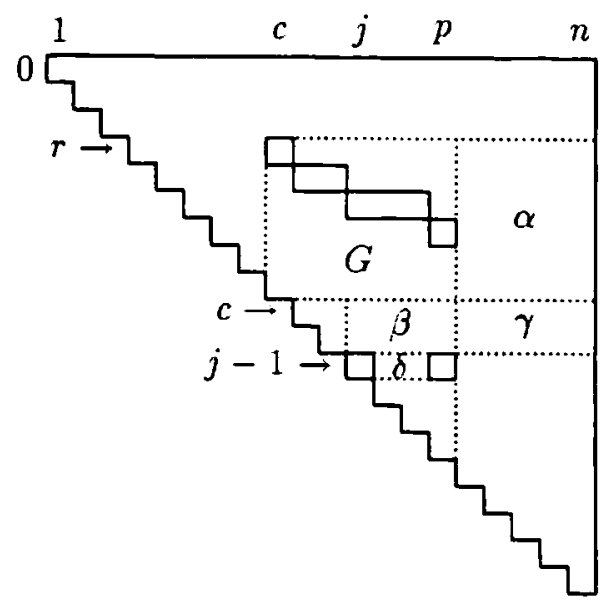

Figure 1. Matrix $B$ at a typical iteration

of the problem proceed simultaneously (i.e., the computation is interleaved among many copies) as in the modified edit distance problem [3] and the mixed convex and concave cost problem [2]. Eppstein [2] extended Wilber's algorithm for interleaved computation. Our algorithm is more general than Eppstein's; it works for any totally monotone matrix $B$ (we use only relation (1)), whereas Eppstein's algorithm works only when $B[i, j]=D[i]+w(i, j)$. Our algorithm is also simpler than both Wilber's and Eppstein's. Recently, Larmore and Schieber [5] reported another linear-time algorithm. which is quite different from ours.

The algorithm consists of a sequence of iterations. Figure 1 shows a typical iteration. We use $N[j], 1 \leq j \leq n$, to store interim column minima before row $r ; N[j]=B[i, j]$ for some $i<r$ (the usage will be clear shortly). At the beginning of each iteration the following invariants hold:

(a) $0 \leq r$ and $r<c$.

(b) $E[j]$ for all $1 \leq j<c$ have been found.

(c) $E[j]$ for $j \geq c$ is $\min \left(N[j], \min _{i \geq r} B[i, j]\right)$.

Invariant (b) means that $D[i]$ for all $0 \leq i<c$ are known, and therefore $B[i, j]$ for $0 \leq i<c$ and $c \leq j \leq n$ is a vailable. Initially, $r=0, c=1$, and all $N[j]$ are $+\infty$.

Let $p=\min (2 c-r, n)$, and let $G$ be the union of $N[c: p]$ and $B[r: c-1, c: p], N[c: p]$ as its first row and $B[r: c-1, c: p]$ as the other rows. $G$ is a $(c-r+1) \times(c-r+1)$ matrix unless $2 c-r>n$. Let $F[j], c \leq j \leq p$, denote the column minima of $G$. Since matrix $G$ is totally monotone, we use the SMAWK algorithm to find the column minima of $G$. Once $F[c: p]$ are found, we compute $E[j]$ for $j=c, c+1, \ldots$ as follows. Obviously, $E[c]=F[c]$. For $c+1 \leq j \leq p$, assume inductively that $B[c: j-2, j: p]$ ( $\beta$ in Figure 1 ) is dead and $B[j-1, j: n]$ is available. It is trivially true when $j=c+1$. By the assumption $E[j]=\min (F[j], B[j-1, j])$.

(1) If $B[j-1, j]<F[j]$, then $E[j]=B[j-1, j]$, and by relation (1) $B[r: j-2, j: n](\alpha, \beta, \gamma$, and the part of $G$ above $\beta$ in Figure 1) and $N(j: n]$ are dead. We start a new iteration with $c=j+1$ and $r=j-1$.

(2) If $F[j] \leq B[j-1, j]$, then $E[j]=F[j]$. We compare $B[j-1, p]$ with $F[p]$.

(2.1) If $B[j-1, p]<F[p], B[r: j-2, p+1: n]$ ( $\alpha$ and $\gamma$ in Figure 1) is dead by relation (1). $B[c: j-2, j: p]$ ( $\beta$ in Figure 1$)$ is dead by the assumption. Thus only $F[j+1: p]$ among $B[0: j-2, j+1: n]$ may become column minima in the future computation. We store $F[j+1: p]$ in $N[j+1: p]$ and start a new iteration with $c=j+1$ and $r=j-1$.

(2.2) If $F[p] \leq B[j-1, p], B[j-1, j: p]$ ( $\delta$ in Figure 1) is dead by relation (1) in submatrix $B[r: j-1, j: p](\beta . \delta$, and the part of $G$ above $\beta)$. Since $B[j, j+1: n]$ is available from 


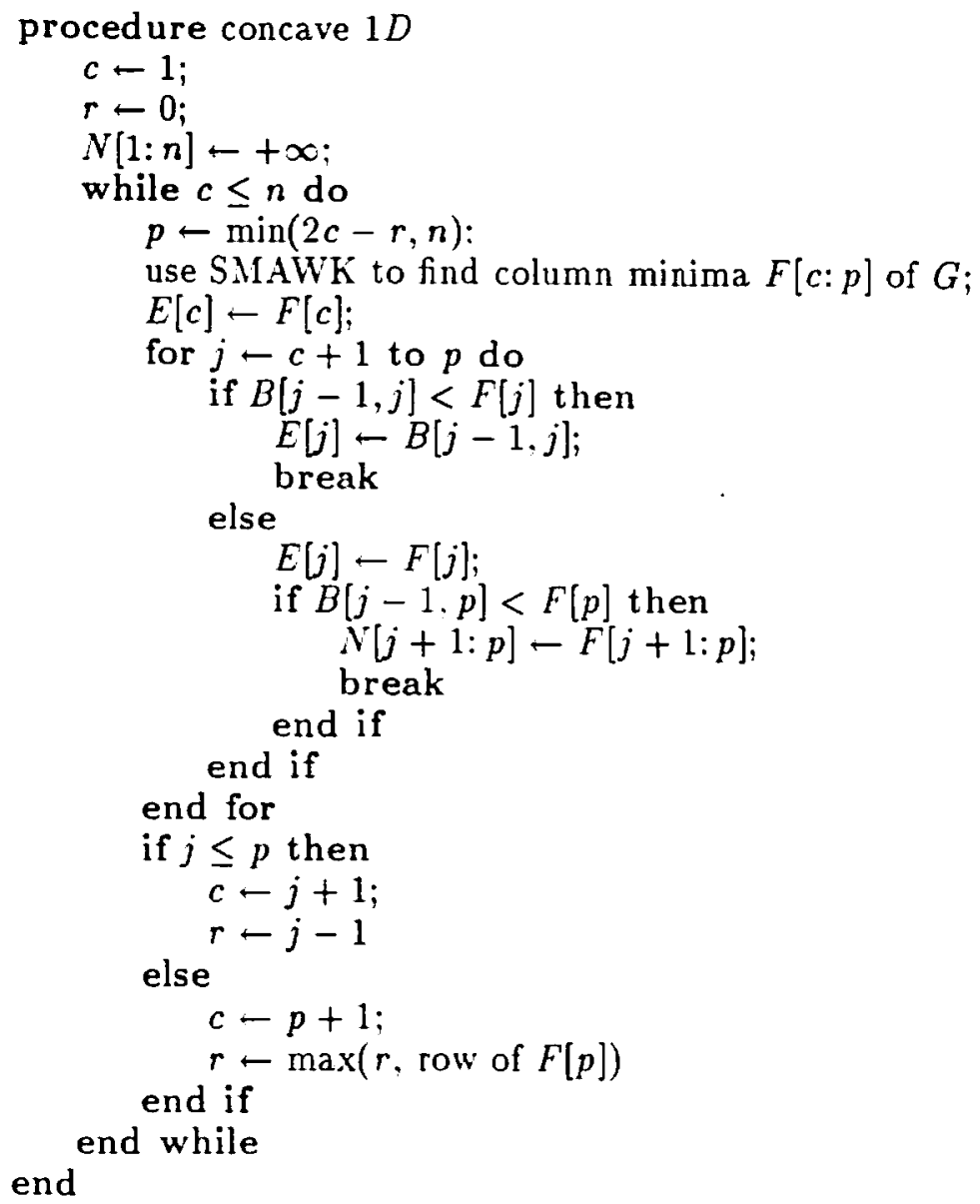

Figure 2. The algorithm for concave $1 D$ dynamic programming

$E[j]$. the assumption holds at $j+1$. We go on to column $j+1$.

If case (2.2) is repeated until $j=p$, we have found $E[j]$ for $c \leq j \leq p$. We start a new iteration with $c=p+1$. If the row of $F[p]$ is greater than $r$, it becomes the new $r$ (it may be smaller than $r$ if it is the row of $N[p]$ ). Note that the three invariants hold at the beginning of new iterations. Figure 2 shows the algorithm, where the break statement causes the innermost enclosing loop to be exited immediately.

Each iteration takes time $O(c-r)$. If either case (1) or case (2.1) happens, we charge the time to rows $r, \ldots, c-1$ because $r$ is increased by $(j-1)-r \geq c-r$. If case (2.2) is repeated until $j=p$, there are two cases. If $p<n$, we charge the time to columns $c \ldots, p$ because $c$ is increased by $(p+1)-c \geq c-r+1$. If $p=n$, we have finished the whole computation, and rows $r, \ldots, c-1(<n)$ have not been charged yet; we charge the time to the rows. Since $c$ and $r$ never decrease, only constant time is charged to each row or column. Thus the total time of the algorithm is linear in $n$. 


\section{References}

[1] Aggarwal, A., Klawe, M. M., Moran, S., Shor, P., and Wilber, R. Geometric applications of a matrix-searching algorithm. Algorithmica 2 (1987), 195-208.

[2] Eppstein, D. Sequence comparison with mixed convex and concave costs. J. Algorithms to appear.

[3] Galil, Z., and Giancarlo, R. Speeding up dynamic programming with applications to molecular biology. Theoretical Computer Science 64 (1989), 107-118.

[4] Hirschberg, D. S., and Larmore, L. L. The least weight subsequence problem. SIAM J. Comput. 16,4 (1987), 628-638.

[5] Larmore, L. L., and Schieber, B. On-line dynamic programming with applications to the prediction of RNA secondary structure. to be presented at the First Annual ACM-SIAM Symposium on Discrete Algorithms.

[6] Wilber, R. The concave least-weight subsequence problem revisited. J. Algorithms 9 (1988), 418-425.

[7] Yao, F. F. Speed-up in dynamic programming. SIAM J. Alg. Disc. Meth. 3 (1982), 532-540. 